Domaines, territoires et frontières en anglais de spécialité

\title{
Leslie Thompson, Jean-Toussaint Pindi, Anglais Appliqué : Sciences du management, Travail, Ressources humaines (master et doctorat) $3^{\mathrm{e}}$ édition
}

Paris : Monchrestien Lextenso éditions, 2012

\section{Séverine Wozniak}

\section{(2) OpenEdition}

\section{Journals}

Édition électronique

URL : http://journals.openedition.org/asp/3802

DOI : 10.4000/asp.3802

ISSN : 2108-6354

\section{Éditeur}

Groupe d'étude et de recherche en anglais de spécialité

\section{Édition imprimée}

Date de publication : 1 novembre 2013

Pagination : 203-205

ISSN : 1246-8185

Référence électronique

Séverine Wozniak, «Leslie Thompson, Jean-Toussaint Pindi, Anglais Appliqué : Sciences du

management, Travail, Ressources humaines (master et doctorat) 3e édition », ASp [En ligne], 64 | 2013,

mis en ligne le 01 novembre 2013, consulté le 02 novembre 2020. URL : http://

journals.openedition.org/asp/3802 ; DOI : https://doi.org/10.4000/asp.3802

Ce document a été généré automatiquement le 2 novembre 2020

Tous droits réservés 


\section{Leslie Thompson, Jean-Toussaint Pindi, Anglais Appliqué : Sciences du} management, Travail, Ressources humaines (master et doctorat) $3^{\mathrm{e}}$ édition

Paris : Monchrestien Lextenso éditions, 2012

Séverine Wozniak

\section{RÉFÉRENCE}

Thompson, Leslie et Jean-Toussaint Pindi. 2012. Anglais Appliqué : Sciences du management, Travail, Ressources humaines (master et doctorat) $3^{\mathrm{e}}$ édition. Paris :

Monchrestien Lextenso éditions, 288 p. ISBN 978-2-7076-1716-3. 
1 Anglais Appliqué: Sciences du management, Travail, Ressources humaines constitue la troisième édition d'un manuel destiné aux étudiants de master et de doctorat en sciences économiques (orientation économie $\mathrm{du}$ travail) et sciences $\mathrm{du}$ management. L'édition précédente datait de 2005 et reprenait le travail initié dans un premier ouvrage intitulé Anglais Appliqué: Gestion, Économie d'entreprise, Travail, Ressources humaines, $2^{e}$ et $3^{e}$ cycles, publié en 2001. Nous notons que les auteurs proposent également chez le même éditeur et dans la même collection («Langues Appliquées», dirigée par l'un des coauteurs du manuel) un ouvrage destiné aux étudiants de licence (Thompson \& Pindi 2009).

Le présent ouvrage s'ouvre avec une

ANGLAIS

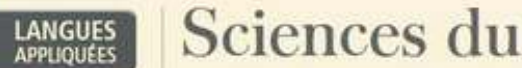
management Theorie Travail

Recherche

Actualité

Etudes de cas

Internet

Exercices

Lexique

Ressources

humaines

Master et Doctorat

Leslie Thompson

Jean-Toussaint Pindi

$3^{e}$ édition

Montchrestien

Monesos introduction de deux pages qui décrit le

dispositif pédagogique mis en œuvre par les auteurs et précise le public ciblé par ce manuel : « Comme dans les éditions précédentes, ce livre trouve son inspiration à la fois dans la notion de genre et dans une démarche de recherche-action. En effet, le discours spécialisé qui est ici didactisé est celui de l'article de recherche relevant des sciences du management» (p. vii). Nous retenons donc que l'ouvrage s'adresse davantage aux doctorants et aux étudiants suivant des formations de master « recherche ». Le manuel se compose de sept parties subdivisées en chapitres; ces parties s'intitulent «Social Issues, Human Resources, Strategy, Customer Relations, Innovation, Business Issues et Projects ".

Chacune des six premières parties comporte un intitulé large ("Social Issues») et des indications pour aider l'étudiant à se repérer : («In this part you will read about... », « You will learn some basic terms concerning... »). Pour amorcer chaque chapitre, une série de mots et de concepts utiles est proposée ${ }^{1}$ : certains sont définis ultérieurement dans le chapitre ; l'étudiant est invité à élucider lui-même le sens d'autres mots et concepts à l'aide d'un dictionnaire. Ensuite, chaque chapitre propose un extrait d'article de recherche traitant du thème général du chapitre. Ces textes, publiés entre 2009 et 2011, sont tous récents. Ils paraissent pour la plupart avoir été écrits par des natifs et permettent d'aborder la question traitée sous l'angle britannique ou/et nordaméricain. Ils font l'objet d'un traitement didactique par le biais d'exercices de compréhension globale et détaillée, de vocabulaire et de grammaire. Enfin des travaux de production, nommés par les auteurs exercices de " contextualisation» (p. viii), sont proposés aux étudiants à la fin de chaque chapitre sous la forme d' "assignments ", conçus pour être exploités par les enseignants dans le cadre de travaux de production écrite, de production ou d'interaction orales (les tâches de compréhension orale ne sont pas incluses dans le dispositif didactique proposé par les auteurs).

La dernière partie, intitulée "Projects", est structurée différemment des autres puisqu'elle est conçue comme une série de tutoriels pratiques sur la rédaction d' 
abstracts et la façon de rendre compte de ce que les auteurs nomment "projets » de recherche (Studying Research Abstract, Reporting on Research, Outlining a Research Article, etc.), d'éléments pratiques (Conducting a Company Case Study) et de recherche (Conducting a Content Analysis). Pour clore ce manuel, les auteurs proposent deux index : l'un porte sur les concepts clés et l'autre rassemble les items grammaticaux abordés au fil des chapitres.

5 Cet ouvrage correspond sans doute assez fidèlement au programme d'un cours de master de management donné par les auteurs. À la lecture des textes sélectionnés, il nous apparaît d'ailleurs que le positionnement disciplinaire est clair et que, contrairement à ce qui est annoncé en introduction, ces documents semblent difficilement exploitables par des étudiants en économie du travail par exemple. De même, il se montre assez peu adapté à des étudiants de master professionnel, tant la dimension "recherche » est mise en évidence. De fait, cet ouvrage s'apparente à un recueil de documents didactisés (type "polycopié »), dont la mise en page quelque peu désuète nous semble avoir peu évolué depuis la première édition. Si la cyberenquête semble a priori incluse dans le dispositif proposé (« Les étudiants sont encouragés, dans la plupart des cas, à rechercher des informations sur Internet afin d'étayer leur argumentation " p. viii), il nous semble qu'il s'agit là d'une déclaration d'intention et que les auteurs pourraient davantage tirer parti de cette possibilité, puisque seuls dix des vingt-quatre chapitres proposent dans leurs assignments ce type de tâche. De même, l'aspect répétitif des exercices proposés ne risque-t-il pas de lasser les étudiants et de poser un problème à l'enseignant d'anglais de spécialité soucieux d'améliorer la dynamique de son cours? Les auteurs ont choisi de ne pas fournir de correction des exercices: cette absence nous semble constituer un point faible pour ce manuel, à l'heure où l'étudiant est appelé à s'impliquer dans son apprentissage notamment à travers le travail en autonomie, plus ou moins guidée par l'enseignant.

6 Toutefois, les textes choisis, tirés de revues reconnues dans le champ des sciences économiques et de gestion, telles que le Journal of Economic Perspectives et le Journal of Economic Issues, ainsi que la California Management Review, la Sloan Management Review et la Harvard Business Review sont indéniablement pertinents. Ils pourront sans doute intéresser des étudiants de master et s'intégrer aisément dans la mise en place d'un dispositif pédagogique de type EMILE, conduit avec les enseignants de spécialité.

\section{BIBLIOGRAPHIE}

Thompson, Leslie et Jean-Toussaint Pindi. 2009. Anglais Appliqué : Économie, Gestion, Droit, AES

(Licence). Paris : Monchrestien Lextenso éditions. 
NOTES

1. À ce sujet, nous avons été gênée par la confusion dans l'introduction entre "terme » (dénomination d'un concept) et « vocabulaire », fût-il « de spécialité » (p. viii).

\section{AUTEURS}

\section{SÉVERINE WOZNIAK}

Université Grenoble Alpes, [ILCEA], F-38040 Grenoble 